\title{
Fractional Quantum Hall Effect States as Exact Ground States
}

\author{
Ranjan Kumar Ghosh \\ Haldia Government College, P.O. Debhog, \\ Midnapore 721657, India \\ Sumathi Rao H \\ Mehta Research Institute, Chhatnag Road,Jhunsi, \\ Allahabad 221506, India
}

\begin{abstract}
We construct many particle Hamiltonians for which the Laughlin and Jain wavefunctions are exact ground states. The Hamiltonians involve fermions in a magnetic field and with inter-particle interactions. For the Laughlin wave-functions, the interactions involve two- and three-body correlations similar to the Chern-Simons interactions, whereas for the projected Jain wavefunctions, $N$-body interactions (which cannot be explicitly written down in general) are involved.
\end{abstract}

PACS numbers:

\footnotetext{
${ }^{1}$ e-mail address: sumathi@mri.ernet.in
} 
The phenomenon of Fractional Quantum Hall Effect (FQHE) [1] continues to hold interest as newer and newer types of heterojunctions are studied at large magnetic fields 22]. It is now well-established that the same kind of correlations are relevant both for the integer (I)QHE and the FQHE and that the phenomenon of FQHE can be understood as IQHE of quasi-particles called composite fermions 3 .

The original Laughlin theory was initiated by a remarkable ansatz wavefunction - the Laughlin wave-function 4 - which worked very well for fractions of the form $1 /(2 m+1)$. Jain recognised that the Laughlin wave-functions could be factorised as a Jastrow factor times the Slater determinant of one filled Landau level (LL). This led him to generalise the Laughlin wavefunctions to the Jain wave-functions [3] by replacing the Slater determinant of one filled LL by that of $n$-filled LLs. This worked for filling fractions of the form $n /(2 m n+1)$, which included all the observed fractions. He interpreted these wave-functions by thinking of the Jastrow factors as even units of flux quanta attached to the electrons. Thus FQHE of electrons at fractions $n /(2 m n+1)$ is equivalent to IQHE of composite electrons (electrons with $2 m$ flux units attached) at level $n$. An apparent drawback of these wave-functions was that they involved fermions in higher LLs, whereas at very high magnetic fields, it is well-known that all the electrons are in the lowest LL (LLL). Quite remarkably, however, it was shown that despite the presence of Slater determinants of higher LLs, the Jain wave-functions are predominantly in the LLL due to the multiplication by Jastrow factors. Moreover, by projecting[5] these wavefunctions onto the LLL, wavefunctions purely in the LLL could be obtained.

There have also been attempts to understand the phenomenon of the FQHE through field theoretic models, where the flux attachment takes place through a Chern-Simons (CS) field. The bosonic field theory approach 6] explains the FQHE at the Laughlin fractions by writing the effective field theory in terms of a bosonic field and showing that the bosonic field Bose condenses at these fractions. The fermionic field theory [7] uses the picture of FQHE as an IQHE of composite fermions à la Jain. However, in both these field theoretic pictures, the flux attachment is through a singular gauge transformation, and at mean field level, only the filling fractions can be determined. The derivation of the actual Laughlin wavefunction is only possible after including fluctuations beyond the mean field picture. More recently, Rajaraman and Sondhi 8 have initiated a field theoretic approach, where the flux 
attachment occurs through a non-unitary transformation. This picture improves upon some of the drawbacks of the earlier field theories and here, in fact, the mean field states are directly the Laughlin states without recourse to fluctuations.

There have been earlier studies of models for which the Laughlin states and Jain states were exact solutions. Models [9, 10] with ultra-short interactions have been shown to have the Laughlin wave-function as an exact ground state whereas a toy model[5] where two Landau levels are exactly degenerate and all higher levels are much higher in energy has the Jain 2/5 state as the exact ground state.

In this letter, we construct explicit models for electrons in an external magnetic field and with specified interactions between particles. When the interactions are of a two-body and three-body type, similar (but not identical) to the CS interaction, we show explicitly that the Laughlin wavefunction is an exact ground state of the system, by rewriting the Hamiltonian in terms of appropriate creation and annihilation operators.

Consider the Hamiltonian given by

$$
\begin{aligned}
& H=\sum_{i}^{N} \frac{\left(\mathbf{p}_{i}-e \mathbf{A}\right)^{2}}{2 m}+\frac{2 \eta}{m} \sum_{i, j \neq i}^{N} \frac{1}{\left(z_{i}-z_{j}\right)}\left(\frac{\partial}{\partial z_{i}^{*}}-\frac{i e A_{z}}{2}\right) \\
& -\frac{2 \eta}{m} \sum_{i, j \neq i}^{N} \frac{1}{\left(z_{i}^{*}-z_{j}^{*}\right)}\left(\frac{\partial}{\partial z_{i}}-\frac{i e A_{z}^{*}}{2}\right)+\frac{2 \eta^{2}}{m} \sum_{i, j \neq i, k \neq i}^{N} \frac{1}{\left(z_{i}-z_{j}\right)\left(z_{i}^{*}-z_{k}^{*}\right)}
\end{aligned}
$$

in two dimensions where $\mathbf{A}=\frac{B}{2}(y,-x)$ is the gauge potential of the external magnetic field, $\left(A_{z}=A_{x}+i A_{y}\right.$ and $\left.A_{z}^{*}=A_{x}-i A_{y}\right), z_{i}=x_{i}+i y_{i}$ denotes the position of the $i^{\text {th }}$ particle and $\eta$ is an odd integer. The second and third terms in the Hamiltonian denote two-body interaction terms whereas the third term denotes a three-body interaction term. We now rescale the distance variable as $z \rightarrow \tilde{z}=z / l$ where $l$ is the magnetic length defined here as $l=1 / \sqrt{e B}$ and rewrite the hamiltonian (dropping the tildes) as

$$
\begin{aligned}
H & =\frac{\omega}{2} \sum_{i}^{N}\left(-4 \frac{\partial^{2}}{\partial z_{i} \partial z_{i}^{*}}+z_{i} \frac{\partial}{\partial z_{i}}-z_{i}^{*} \frac{\partial}{\partial z_{i}^{*}}+\frac{z_{i} z_{i}^{*}}{4}\right) \\
& +\omega\left[2 \eta \sum_{i, j \neq i}^{N} \frac{1}{\left(z_{i}-z_{j}\right)}\left(\frac{\partial}{\partial z_{i}^{*}}-\frac{z_{i}}{4}\right)-2 \eta \sum_{i, j \neq i}^{N} \frac{1}{\left(z_{i}^{*}-z_{j}^{*}\right)}\left(\frac{\partial}{\partial z_{i}}+\frac{z_{i}^{*}}{4}\right)\right.
\end{aligned}
$$




$$
\left.+2 \eta^{2} \sum_{i, j \neq i, k \neq i}^{N} \frac{1}{\left(z_{i}-z_{j}\right)\left(z_{i}^{*}-z_{k}^{*}\right)}\right]
$$

where $\omega=e B / m$.

Now let us consider the action of this Hamiltonian on the Laughlin wavefunction given by

$$
\psi_{L}=\prod_{i<j}^{N}\left(z_{i}-z_{j}\right)^{\eta} e^{-\sum_{i} \frac{z_{i} z_{i}^{*}}{4}} \equiv f^{\eta} e^{-\sum_{i} \frac{z_{i} z_{i}^{*}}{4}} .
$$

We use the fact that $\frac{\partial}{\partial z_{i}} \ln f=\sum_{j \neq i} \frac{1}{\left(z_{i}-z_{j}\right)}$ and $\frac{\partial}{\partial z_{i}^{*}} \ln \bar{f}=\sum_{j \neq i} \frac{1}{\left(z_{i}^{*}-z_{j}^{*}\right)}$, (where $\bar{f}$ is the complex conjugate of $f$ ) to obtain

$$
H \psi_{L}=\frac{N \omega}{2} \psi_{L}
$$

Thus, we find that the Laughlin wave-function is an exact eigenstate of the Hamiltonian in Eq.(1) with eigenvalue $N \omega / 2$. To prove that $\psi_{L}$ is a ground state, following a general procedure 11, we rewrite the Hamiltonian in terms of creation and annihilation operators

$$
\begin{aligned}
a_{i} & =\frac{1}{\sqrt{2}}\left(2 \frac{\partial}{\partial z_{i}}-2 \eta \frac{\partial}{\partial z_{i}} \ln f+\frac{z_{i}^{*}}{2}\right) \\
\text { and } \quad a_{i}^{\dagger} & =\frac{1}{\sqrt{2}}\left(-2 \frac{\partial}{\partial z_{i}^{*}}-2 \eta \frac{\partial}{\partial z_{i}^{*}} \ln \bar{f}+\frac{z_{i}}{2}\right)
\end{aligned}
$$

as

$$
H=\omega \sum_{i}^{N} a_{i}^{\dagger} a_{i}+\frac{N \omega}{2} .
$$

It is easy to check that $a_{i}$ for all $i$ annihilates the state defined by the Laughlin wave-function, which is hence the ground state of this Hamiltonian with the energy $N \omega / 2$. (An equation similar to $a_{i} \psi=0$ has been studied earlier in the context of anyons [12]). Moreover, $a_{i}$ and $a_{i}^{\dagger}$ satisfy the canonical harmonic oscillator commutation rules even for non-zero $\eta$. Hence, the Hamiltonian is exactly soluble for all the excited states.

\footnotetext{
${ }^{2}$ We ignore the term $\sum_{j \neq i} \delta\left(z_{i}-z_{j}\right)$ obtained by acting $\frac{\partial}{\partial z_{i}^{*}}$ on $\frac{\partial}{\partial z_{i}} \ln f$ since it is irrelevant when acting on antisymmetric wave-functions.
} 
Let us now interpret the interaction terms in the Hamiltonian. By defin$\operatorname{ing} F=(\ln f-\ln \bar{f})$, and introducing a new vector $(\mathrm{NV})$ potential $\mathbf{V}_{i}^{L}$ at the position of the $i^{\text {th }}$ particle as

$$
\mathbf{V}_{i}^{L}=-i \eta \vec{\nabla}_{i} F=-i \eta \vec{\nabla}_{i}(\ln f-\ln \bar{f})
$$

we see that the interactions implied by the two- and three-body can be interpreted as a gauge potential. The gauge potential can be equivalently written in complex notation as

$$
\begin{aligned}
V_{i z}^{L} & =-2 i \eta \sum_{j \neq i}^{N} \frac{1}{\left(z_{i}-z_{j}\right)}=-2 i \eta \frac{\partial}{\partial z_{i}} \ln f=-2 i \eta \frac{\partial}{\partial z_{i}}(\ln f-\ln \bar{f}) \\
\text { and } V_{i z}^{L *} & =-2 i \eta \sum_{j \neq i}^{N} \frac{1}{\left(z_{i}^{*}-z_{j}^{*}\right)}=-2 i \eta \frac{\partial}{\partial z_{i}^{*}} \ln \bar{f}=2 i \eta \frac{\partial}{\partial z_{i}^{*}}(\ln f-\ln \bar{f}),(8)
\end{aligned}
$$

(since $2 \frac{\partial}{\partial z_{i}}=\frac{\partial}{\partial_{x}}-i \frac{\partial}{\partial y}$ and $2 \frac{\partial}{\partial z_{i}^{*}}=\frac{\partial}{\partial x}+i \frac{\partial}{\partial y}$ ). In terms of this gauge potential, we find that the original Hamiltonian in Eq.(四) can be rewritten as

$$
H=\sum_{i}^{N} \frac{\left(\mathbf{p}_{i}-e \mathbf{A}-\mathbf{V}_{\mathbf{i}}\right)^{2}}{2 m} .
$$

Note that $\mathbf{V}_{i}$ is purely real, so that there is no problem in interpreting it as a vector potential. It is now clear that this Hamiltonian can be obtained by a singular gauge transformation from the 'free' Hamiltonian, with no interparticle interactions given by

$$
H_{0}=\sum_{i}^{N} \frac{\left(\mathbf{p}_{i}-e \mathbf{A}\right)^{2}}{2 m} .
$$

However, there are several subtleties to note here. One is that $H_{0}$ has antianalytic wave-functions as the lowest energy (LLL) wave-functions. Under the transformation

$$
\begin{aligned}
\psi^{*} \rightarrow \psi^{\prime} & =e^{\eta(\ln f-\ln \bar{f})} \psi^{*}=\prod_{i<j}^{N}\left(\frac{\left(z_{i}-z_{j}\right)}{\left(z_{i}^{*}-z_{j}^{*}\right)}\right)^{\eta} \psi^{*} \\
\mathbf{V}_{i}^{L} \rightarrow \mathbf{V}_{i}^{L^{\prime}} & =0+\eta \vec{\nabla}_{i} \sum_{j \neq i}^{N} \ln \frac{\left(z_{i}-z_{j}\right)}{\left(z_{i}^{*}-z_{j}^{*}\right)}
\end{aligned}
$$


the 'free' Hamiltonian in Eq.(10) gets transformed to the interacting Hamiltonian in Eq.(9). Secondly, without the Coulomb interaction, the Laughlin wave-function is not the unique ground state either of the original 'free' Hamiltonian or the gauge transformed Hamiltonian. However, we follow the general procedure adopted in the Chern-Simons literature and assume that the Coulomb interactions will pick the Laughlin state to have the lowest energy. The aim of all the vector potential models is more to identify new mean field theories and new order parameters.

To get a clearer understanding of this potential, since it depends on the positions of other particles in the system, it is natural to compare it with the CS gauge potential which also arises due to the presence of other particles. Traditionally, the CS potential is written in cylindrical coordinates as

$$
\mathbf{a}_{i}^{\mathrm{CS}}=(\eta-1) \sum_{j \neq i}^{N} \frac{\hat{z} \times\left(\mathbf{r}_{i}-\mathbf{r}_{j}\right)}{\left|\mathbf{r}_{i}-\mathbf{r}_{j}\right|^{2}}
$$

where $\mathbf{r}_{i}$ and $\mathbf{r}_{j}$ are the position vectors of the particles and we are considering fermion to fermion transformation. However, it can also be written as

$$
\mathbf{a}_{i}^{\mathrm{CS}}=(\eta-1) \vec{\nabla}_{i} \sum_{j \neq i}^{N} \theta_{i j}
$$

where $\theta_{i j}$ is the relative angle between the position vectors of the $i^{\text {th }}$ and $j^{\text {th }}$ particles. It can be generated from the Hamiltonian of particles in a uniform magnetic field

$$
H_{0}=\sum_{i}^{N} \frac{\left(\mathbf{p}_{i}+e \mathbf{A}\right)^{2}}{2 m}
$$

(note the change in sign of $B$ ), with the magnetic field adjusted so that the filling factor is $1 / \eta$ by a singular gauge transformation, at the expense of attaching phase factors to the wave-function. Under the transformation

$$
\begin{aligned}
\psi \rightarrow \psi^{\prime} & =e^{i(\eta-1) \sum_{j \neq i}^{N} \theta_{i j}} \psi=\prod_{i<j}^{N}\left(\frac{\left(z_{i}-z_{j}\right)}{\left|z_{i}-z_{j}\right|}\right)^{(\eta-1)} \psi \\
\mathbf{a}_{i} \rightarrow \mathbf{a}_{i}^{\prime} & =\mathbf{a}_{i}-(\eta-1) \vec{\nabla}_{i} \sum_{j \neq i}^{N} \theta_{i j}=0-i(\eta-1) \vec{\nabla}_{i} \sum_{j \neq i}^{N} \ln \frac{\left(z_{i}-z_{j}\right)}{\left|z_{i}-z_{j}\right|}
\end{aligned}
$$


In this case, both the original and gauge transformed Hamiltonians have analytic wave-functions as LLL states.

The Chern-Simons potential was introduced in the context of FQHE as an exact transformation on the Hamiltonian for particles in an external magnetic field and interacting through a Coulomb potential. A mean field solution of the transformed Hamiltonian is then shown to yield the correct filling factors and the Laughlin wave-function as a solution of the model is only obtained after including fluctuations in the random phase approximation.

Now, let us compare our NV potential with the CS potential. Since $\mathbf{V}_{i}^{L}$ can be written as

$$
\mathbf{V}_{i}^{L}=-i \eta \vec{\nabla}_{i} \ln \frac{f}{\bar{f}}=2 \eta \vec{\nabla}_{i} \sum_{j \neq i}^{N} \theta_{i j}
$$

we see that it is precisely the Chern-Simons potential, except that it interacts with a strength $2 \eta$ instead of $(\eta-1)$. So, we seem to be led to the result that the Hamiltonian for which the Laughlin wave-function is an exact ground state interacts with a Chern-Simons field with a different charge than the usual Chern-Simons theory, where the Laughlin wave-function is not an exact solution, but appears after performing the mean field approximation and then including fluctuations.

However, this puzzle can be understood in the following way. As we mentioned before, when the interaction terms are switched off in Eq.(5), $a_{i}$ annihilates anti-analytic wave-functions, which, in turn implies that the sign of the magnetic field has been switched. This leads us to the following scenario. We start with the non-interacting Hamiltonian in Eq.(14) with the magnetic field adjusted so that the filling factor is $1 / \eta$. The usual Chern-Simons procedure redistributes the total flux ( $\eta$ per particle) by decreasing the magnetic field to unit flux per particle and localising the remaining flux $((\eta-1)$ per particle), at the position of the particles. Since $(\eta-1)$ is an even integer, $(\eta$ is odd), fermions are converted to composite fermions which see the appropriate magnetic field for integer QHE. This is the mean field approximation, where the filling fractions are obtained correctly but the wave-function is wrong (it is, in fact, singular). Only when we include fluctuations about the mean field, can we correctly obtain the Laughlin wave-function as the ground state.

But the total flux $\eta$ can also be redistributed in the following way. We may have a uniform magnetic field with $-\eta$ flux per particle (equivalent to chang- 
ing the sign of the external magnetic field and obtaining the free Hamiltonian in Eq.(10) instead of Eq.(14)) and localise $2 \eta$ units of flux per particle, thus converting them to a different kind of composite fermion. In other words, the Hamiltonian that we have written down in Eq.(1) is equivalent to the Hamiltonian in Eq.(14), which is appropriate for the FQHE, at a mean field level. The interesting feature of this picture is that at the mean field level itself, the Laughlin wave-function emerges as an exact ground state.

However, we should point out that this scenario does not lead to a particularly useful mean field approximation. As compared to the original Hamiltonian in Eq.(14) plus the Coulomb interaction, the Hamiltonian in Eq.(11) is not really simpler. Its only virtue is that it can be solved exactly for the ground state.

For completeness, we also compare our vector potential with yet another vector potential in the literature discussed by Rajaraman and Sondhi[8]. A quantum mechanical analogue of their field theory involves starting with the non-interacting Hamiltonian in Eq.(14) and making a non-unitary transformation on the wave-function as

$$
\psi \rightarrow \psi^{\prime}=e^{(\eta-1) \ln f} \psi=\prod_{i<j}^{N}\left(z_{i}-z_{j}\right)^{(\eta-1)} \psi
$$

for a fermion to fermion transformation. (Note that the transformation function is purely analytic, in contrast to the transformations in Eqs(11) and (16).) Under this transformation, the usual harmonic oscillator creation and annihilation operators transform as

$$
b_{i}=\frac{1}{\sqrt{2}}\left(-2 \frac{\partial}{\partial z_{i}^{*}}+\frac{z_{i}}{2}\right) \rightarrow b_{i}^{\prime}=b_{i}
$$

(since $\frac{\partial}{\partial z_{i}^{*}} \ln f=0$ ) and

$$
b_{i}^{\dagger}=\frac{1}{\sqrt{2}}\left(2 \frac{\partial}{\partial z_{i}}+\frac{z_{i}^{*}}{2}\right) \rightarrow b_{i}^{\dagger}=\frac{1}{\sqrt{2}}\left(2 \frac{\partial}{\partial z_{i}}+2(\eta-1) \frac{\partial}{\partial z_{i}} \ln f+\frac{z_{i}^{*}}{2}\right) .
$$

The Hamiltonian is then constructed as

$$
H=\sum_{i}^{N} b_{i}^{\prime \dagger} b_{i}+\frac{N \omega}{2} .
$$


We identify $V^{C}=-i(\eta-1) \vec{\nabla}_{i} \ln f$ as a complex vector $(\mathrm{CV})$ potential. But for the inessential difference that the vector field introduced in Ref. [8] includes the constant external magnetic field as well, this CV field (upto a phase redefinition) is the same vector field introduced by them in a field theoretic context.

Thus, under the transformation on the wave-function given in Eq.(18) accompanied by the following transformation on the complex vector field,

$$
V^{C} \rightarrow V^{C^{\prime}}=0-i(\eta-1) \sum_{j \neq i}^{N} \ln \left(z_{i}-z_{j}\right),
$$

the non-interacting Hamiltonian in Eq.(14) gets precisely transformed to the interacting Hamiltonian in Eq.(21). Note that the the Hamiltonian in Eq.(21) is not naively hermitean. However, by defining a new inner product in the Hilbert space

$$
<\psi|O| \phi>=\int \psi^{*} O \phi e^{-(\eta-1)(\ln f+\ln \bar{f})} d^{2} x
$$

analogous to the field redefinitions made in Ref. [8], the Hamiltonian becomes hermitean because

$$
\int \psi^{*}\left(b_{i} \phi\right) e^{-(\eta-1)(\ln f+\ln \bar{f})} d^{2} x=\int\left(b_{i}^{\dagger} \psi\right)^{*} \phi e^{-(\eta-1)(\ln f+\ln \bar{f})} d^{2} x .
$$

Hence, the Rajaraman-Sondhi Hamiltonian is hermitean although it has been obtained by a non-unitary transformation.

The CV field attaches 'fat' vortices to fermions, unlike the CS gauge field which attaches infinitesimal flux-tubes, where only the phase is relevant. This is closer to Jain's picture, where he attaches 'fat' vortices to electrons to turn them into composite fermions. The 'fat' vortex factors increase the degree of the polynomial and consequently, the area occupied by the particles in the circular droplet. However, the magnetic field $B$ and the total number of particles $N$ remain fixed. This means that the density of the particles has decreased. Hence, adding 'fat' vortex factors changes the filling fraction and is not a gauge transformation.

The CV field has a representation in terms of the CS gauge field. In fact, it is easy to check from the explicit definitions of the CS field and the CV field that

$$
\mathbf{V}^{C}=i \mathbf{a}^{\mathrm{CS}}+\hat{z} \times \mathbf{a}^{\mathrm{CS}}
$$


Thus, unlike the CS field which had only angular components, the CV field has both radial and angular components. Note however, that the density dependence of the CV field is solely through the CS construction.

Since the Laughlin wave-function is the exact ground state wave-function of the Hamiltonian with particles interacting through the $\mathbf{V}_{i}^{L}$ field with a strength proportional to the inverse of the filling fraction, the bottomline question to address is why the Coulomb interaction between particles disguise themselves as these vector interactions. We have no insight into this problem.

What we have instead addressed are the differences between attaching flux-factors $\Pi_{i<j}^{N} \frac{\left(z_{i}-z_{j}\right)}{\left|z_{i}-z_{j}\right|}$ through the CS gauge field, the factor $\prod_{i<j}^{N} \frac{\left(z_{i}-z_{j}\right)}{\left(z_{i}^{*}-z_{j}^{*}\right)}$ through the NV field, and the vortex factor $\prod_{i<j}^{N}\left(z_{i}-z_{j}\right)$ through the CV field. Thus, the NV and CV Hamiltonians also (and correspondingly the appropriate field theories) deserve further study to see if they yield quantitatively correct results.

Is it possible to construct new Hamiltonians for which the projected Jain wave-functions are exact ground states? The projected Jain wave-functions are given by

$$
\psi_{J}=f_{J} e^{-\sum_{i} \frac{z_{i} z_{i}^{*}}{4}}=\mathrm{P} \prod_{i<j}^{N}\left(z_{i}-z_{j}\right)^{2 m} \chi_{n} e^{-\sum_{i} \frac{z_{i} z_{i}^{*}}{4}} .
$$

where $\mathrm{P}$ is the projection operator that projects the wave-function onto the LLL. This means that to get the actual form of $f_{J}$, we need to make the substitution $z_{i}^{*} \rightarrow 2 \frac{\partial}{\partial z_{i}}$ (with all $z^{*}$ 's to the left of the $z$ 's before substitution) 司. Thus, $f_{J}$ is an analytic function of the $z_{i}$ 's with terms of the form

$$
z_{1}^{r_{1}} z_{2}^{r_{2}} \cdots z_{N}^{r_{N}}
$$

with coefficients which can be explicitly determined by expanding the Jastrow factor, the determinant and by acting with the derivatives. In practice, this is only possible for small values of $N$. However, even in general, $f_{J}$ is a homogeneous function of its $N$ variables, since both $\chi_{n}$ and the Jastrow factor are homogeneous. Moreover, as we have already mentioned, the projection operator $\mathrm{P}$ ensures that $f_{J}$ is analytic. For a homogeneous and analytic $f_{J}$, we find that the wave-function $\psi_{J}$ is a ground-state of the same Hamiltonian in Eq.(6) with $a_{i}$ and $a_{i}^{\dagger}$ still given by Eq.(可). However, now the interaction term $\frac{\partial}{\partial z_{i}} \ln f_{J}=\frac{\partial}{\partial z_{i}}\left(\ln f_{J}-\ln \bar{f}_{J}\right)$ can have arbitrary $N$-body interactions, since 
$f_{J}$ is an arbitrary homogeneous and analytic function. We may still interpret the interaction term as a vector potential $V_{i z}^{J}=2 i \frac{\partial}{\partial z_{i}}\left(\ln f_{J}-\ln \bar{f}_{J}\right)$, but this is no longer related to the CS gauge field $\mathbf{a}_{i}^{\mathrm{CS}}$ like $\mathbf{V}_{i}^{L}$. More specifically, $\mathbf{V}_{i}^{J}$ is not constrained by the density of particles alone, but by more complicated correlations. This explains why these states have not been amenable to the usual CS - type field theoretic treatments, although they have the virtue of analyticity. Much further work is needed to understand the nature of this kind of NV potential.

In conclusion, in this letter, we have shown that the Laughlin wavefunction is an exact ground state of a Hamiltonian with many particles in an external magnetic field and with inter-particle interactions that can be described by a NV field related to the density of particles. We have also compared our vector potential with the Chern-Simons and Rajaraman-Sondhi vector potentials and discussed the merits and demerits of the different approaches.

\section{Acknowledgments}

One of us (S.R.) would like to thank D. M. Gaitonde and R. Rajaraman for useful discussions. RKG would like to thank the Mehta Research Institute for hospitality during the initiation of this work.

\section{References}

[1] D. C. Tsui, H. L. Stormer and A. C. Gossard, Phys. Rev. Lett. 48, 1559 (1992); The Quantum Hall Effect, edited by R. E. Prange and S. M. Girvin, Springer -Verlag, New York, 1987; The Fractional Quantum Hall Effect, Springer series in Solid State Sciences, 85, Springer-Verlag, 1988.

[2] H. L. Stormer et al, Phys. Rev. Lett.bf 56, 85 (1986); G. S. Boebinger et al, Phys. Rev. Lett.64, 1793 (1990); Y. W. Suen et al, Phys. Rev. Lett.68, 1379 (1992); J. P. Eisenstein et al, Phys. Rev. Lett.68, 1383 (1992); S. Q. Murphy et al, Phys. Rev. Lett.72, 728 (1994). 
[3] J. K. Jain, Phys. Rev. Lett. 63, 199 (1984); V. J. Goldman, B. Su and J. K. Jain, Phys. Rev. Lett. 72, 2065 (1994).

[4] R. B. Laughlin, Phys. Rev. Lett. 50, 1395 (1983).

[5] J. K. Jain, Advances in Physics, 41, 105 (1992); G. Dev and J. K. Jain, Phys. Rev. B45, 1223 (1992)

[6] S. C. Zhang, T. H. Hannson, and S. Kivelson, Phys. Rev. Lett. 62, 82 (1989); S. C. Zhang, Int. J. Mod. Phys. B 6, 25 (1992).

[7] A. Lopez and E. Fradkin, Phys. Rev. B44, 5246 (1991); see also E. Fradkin, Field Theories of Condensed Matter Systems, Addison-Wesley, Redwood City, 1991.

[8] R. Rajaraman and S. L. Sondhi, Int. J. of Mod. Phys. B 10, 793 (1996).

[9] F. D. M. Haldane, Phys. Rev. Lett. 51, 605 (1983).

[10] S. A. Trugman and S. Kivelson, Phys. Rev. B31, 5280 (1985).

[11] R. K. Ghosh and S. Rao, Exactly Solvable Models in Arbitrary Dimensions, MRI preprint, MRI-PHY/P9704009

[12] Z. F. Ezawa, M. Hotta and I. Iwazaki, Phys. Rev. B46, 7765 (1992). 